\title{
Growth and Development of Continuous Structures
}

\author{
Or Yogev \\ California Institute of Technology \\ 1200 East California Blvd. \\ Pasadena, CA 91125 U.S.A. \\ or@caltech.edu
}

\author{
Erik K. Antonsson \\ California Institute of Technology \\ 1200 East California Blvd. \\ Pasadena, CA 91125 U.S.A. \\ erik@design.caltech.edu
}

\begin{abstract}
Nature has demonstrated the capability to evolve high performance systems. In natural evolution, genetic information is used to control the growth and development of individuals, whose performance in the environment determines whether their genetic information is passed on to subsequent generations. In this paper an artificial biological model has been created. The model uses artificial genetic information to control the growth and development of continuous inhomogeneous structures, where sets of rules control the growth and development of the individual. Using this method a relatively simple artificial genome can give rise to highly complex structures without defining explicitly the final configuration and form convenient model for evolution.
\end{abstract}

\section{Categories and Subject Descriptors}

J.2 [Physical Sciences and Engineering]: Engineering

\section{General Terms}

Design

\section{Keywords}

Genetic Algorithm, Indirect Encoding, Stresses, Finite Elements, Artificial Cell

\section{ARTIFICIAL CELLS}

The artificial cell introduced here represents an extended three-dimensional linear hexahedral finite element. Each cell can sense two types of quantities which control growth and development of the structure, one refers to mechanical stress and the other refers to the morphogen diffusion level. These two qualities have proven to have crucial effects on the growth of organisms $[1,2]$. The stresses refer to the point inside the cell with the maximum principal stress. The three principal stresses are normalized by the yield stress of the material, and are identified in the genome with the letters $a, b, c$. Their corresponding principal directions are identified in the genome with the letters $d, e, f$. There are two kinds of morphogens, one is a morphogen corresponding to a point load in space that is to be supported; the other is a morphogen corresponding to the ground. Each morphogen diffuses through space through the cell walls with

Copyright is held by the author/owner(s).

GECCO'07, July 7-11, 2007, London, England, United Kingdom.

ACM 978-1-59593-697-4/07/0007.

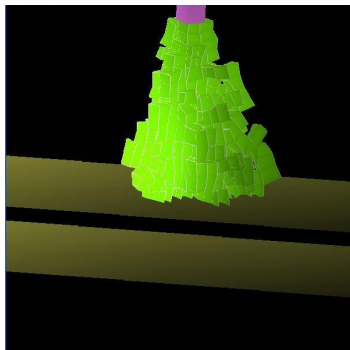

a

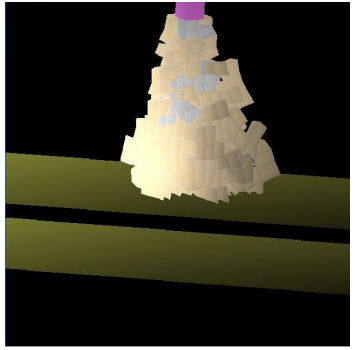

$\mathrm{b}$
Figure 1: The two images show the final configuration of the structure resulting from the execution of the genome sequence. The colors in a) represent mechanical stresses where green indicates stresses that are much less then the yield stress. The colors in b) represent different mechanical properties.

a corresponding direction and intensity (gradient). These two quantities are identified in the genome with the letters $h$ and $i$ respectively. Rules (or words) are composed of genes. The model contains three kinds of genes. The first are conditional genes $V, W$ which suppress gene activity. The second and the third are operation or transformation genes. Operation genes correspond to geometrical operations which change the shape of the cell $(C, B, S)$, and cell-type operations which divide or kill cells $(D, K)$. Transformation genes change the mechanical properties of the cell $(F)$.

\section{RESULTS}

The model has been tested with two signaling morphogens. One represents the ground $(20 m \times 20 m \times 5 m)$, and the other represents a load - $(15,000 \mathrm{kN}$ in the $x$ direction, $15 \mathrm{~m}$ above the center (see Figure 1)). The test genome code consists of five words, and produces a single individual structure. The genome contains the following code:

Z5C1h Z4A1i Z3V2aB5a5b5c Z2W1aB1a1b1c Z1W99gDidef

\section{REFERENCES}

[1] Lars Hufnagel, Aurelio A. Teleman, H. R., 2007. "On mechanism of wing sice determination in fly development". PNAS, 104(10), March, pp. 3835-3840.

[2] Dickinson, M. H., 1999. "Bionics: Biological insight into mechanical design". PNAS, 96(25), pp. 14208-14209. 